\title{
Analyse ergonomique de l'activité enseignante au cours d'un cycle de football, en milieux « ordinaire » et « difficile » avec débat d'idées
}

\author{
Gharbi Maher
}

Gharbi Maher.

Résumé. Cette recherche vise à analyser l'activité de deux enseignants ayant participé à une interventionrecherche en ergonomie de l'activité. Exerçant dans deux collèges différents de l'éducation prioritaire et ordinaire, ils se sont retrouvés face à la mise en place d'un artefact verbal durant les quatre séances d'enseignement de football qu'ils ont assurées chacun de leur côté. Grâce au cadre de l'intervention-recherche, nous analyserons l'activité de chacun des deux enseignants suite à l'installation du débat d'idées comme un outil didactique en leur faveur. La mise en place des auto-confrontations simples et croisées comme méthodologie de recueil de donnée, nous a permis de montrer l'importance donnée par les enseignants pour ce type d'artefact verbal mais aussi le poids qu'il génère sur leurs activités ce qui est expliqué par sa mise à l'écart durant les cours.

Mots-clés. Ergonomie de l'activité, Intervention-recherche, Football, Milieu ordinaire et difficile, Débat d'idées

\section{Introduction}

La présente recherche tente d'observer et d'analyser l'activité enseignante, entre préoccupations et occupations, dans le cadre d'un cycle de football en collège. Elle a pour terrain une session d'enseignement de football proposée à des élèves de cycle 4 ( $\left.4^{\text {ème }}\right)$ par deux enseignants. Ces derniers exercent leur métier dans un milieu scolaire ordinaire et prioritaire.

«Enseigner est un métier » 1 : ce constat est issu des réflexions de plusieurs chercheurs (Saujat, 2002) dont nous faisons partie. L'idée est qu'un enseignant ne peut pas s'appuyer uniquement sur ses savoirs académiques ; il doit aussi disposer d'une formation complémentaire lui apportant des compétences didactiques et pédagogiques.

Au cours des 30 dernières années, l'EPS a connu des mutations institutionnelles profondes, officialisées par la parution de nombreux textes organisant la discipline. Si l'on part du principe que l'éducation physique et sportive est une matière particulière seulement « dans, par et pour l'école » (Arnaud, 1989), les enseignants sont donc amenés, au-delà de leurs appartenances à différents milieux, à construire leur identité professionnelle dans une relation spécifique avec cette discipline scolaire et les élèves.

De ce fait, l'activité des enseignants d'EPS est appréhendée dans des situations qui ont pour objectifs de mobiliser et d'entretenir le collectif de travail et au travail que constituent les élèves (Saujat \& Serres, 2015). Dans la lignée de ce qui a été dit précédemment, Bruner et Bonin (1996) évoque les objets de recherche de nombreux pédagogues concernant l'activité des élèves et précise qu'«il ne suffit pas de décrire ce que fait l'enfant; ce qui est désormais à l'ordre du jour, c'est de déterminer ce qu'il pense être en train de faire et pour quelles raisons il le fait » (p. 69). La présente recherche s'inscrit dans les réflexions précédentes. Même si de telles préoccupations ne sont pas nouvelles, elle tente d'apporter un regard original sur les enseignants, entre ce qu'ils veulent faire, ce qu'ils devraient faire et ce qu'ils ont fait réellement (Clot, Faïta, Fernandez \& Scheller, 2000). Le point de départ est la mise en œuvre d'un cycle de football avec débat d'idées dans le cadre des APSA ; il faut souligner que ce type de projet est rarement programmé par les enseignants d'EPS. 
Analyse ergonomique de l'activité enseignante au cours d'un cycle de football, en milie...

\section{Méthodologie}

\section{La difficulté de départ : le débat d'idées, un instrument et/ou un dilemme}

La communauté des ergonomes considère comme une «règle de métier » le fait « de comprendre le travail pour le transformer, à la demande des intéressés » (Laurent et Saujat, 2014). Dans une même démarche, Wisner (1995) explique que « la plupart des chercheurs en sciences humaines n'aiment pas obéir à une commande. Nous, au contraire, considérons la demande comme absolument essentielle en ergonomie parce qu'elle nous conduit sur la difficulté » (p. 10).

La difficulté qui est à l'origine de notre travail ne découle pas des professionnels du métier, qu'il s'agisse des enseignants d'EPS faisant face à de multiples contraintes ou des enseignants qui essayent de s'adapter à leur milieu de travail $\underline{2}$. Elle émane plutôt de l'introduction d'un artefact $\underline{3}$ datant de 1998, qui est rarement mis en place à travers une pratique $\underline{4}$ souvent utilisée mais rarement enseignée (Roy \& Tessier, 2006) dans des milieux différents.

Cet artefact (Rabardel, 1995) qui est traité uniquement du point de vue didactique depuis son apparition, nous le projetons dans le milieu ergonomique pour savoir comment il devient ou non un instrument dans l'activité et observer son évolution dans cette activité pour chacun des deux enseignants participants. Nous nous appuyons ici sur les travaux de Rabardel (1995) pour expliquer notre propos, à travers les deux processus d'instrumentation et d'instrumentalisation. C'est-à-dire que nous cherchons à savoir comment les enseignants ont pris en main le débat d'idées et comment ils se l'approprient durant les séances.

\section{Le déroulement de l'intervention-recherche}

Cette intervention-recherche a débuté durant l'année scolaire 2017-2018 et s'est achevée en décembre 2019. Elle concerne deux enseignants exerçant dans deux classes du même cycle mais de milieux différents $\underline{5}$. Le premier recueil de données a été effectué au cours de l'année scolaire 2017-2018, dans le collège Edmond Rostand de l'académie d'Aix-Marseille.

C'est un collège urbain situé dans le $13^{\text {ème }}$ arrondissement de Marseille et appartenant au réseau d'éducation prioritaire renforcé (REP+). Le recueil des données a été mené avec une classe de $4^{\text {ème }}$ dans un cycle de football. Cette classe comprend 23 élèves présentant des difficultés scolaires et sociales. Toutefois, pour Thomas $\underline{6}$, leur enseignant, c'est une classe dynamique, qui a un bon comportement sur le terrain, malgré certains problèmes. Plusieurs élèves, dont une fille, jouent déjà au football dans un cadre civil. Il est important aussi de préciser que cette classe est composée d'une majorité d'élèves nés en France mais dont les parents ont des origines différentes.

Le deuxième recueil de données a été effectué également durant l'année scolaire 2017-2018 mais dans un collège urbain de la région niçoise: La Bourgade. Contrairement au précédent, il ne fait pas partie des zones d'éducation prioritaire (ZEP) ; c'est un collège ordinaire de l'académie de Nice. Quant à la classe étudiée, les données fournies par l'équipe éducative des enseignants d'EPS montrent qu'elle est composée de 25 élèves, qu'elle est dynamique, sans difficulté scolaire apparente, avec un taux de réussite élevé.

\section{Une intervention instrumentée}

La première étape est un temps pour agir auprès des enseignants, instrumentée par la méthode des autoconfrontations simples et croisées (Faïta \& Viera, 2003). Chaque enseignant a été filmé en exerçant son activité en s'intéressant aux situations de mise en place ou non du débat d'idées puis confronté aux images de ces situations initiales lors d'une autoconfrontation simple (ACS) entre le chercheur et l'enseignant puis croisée entre les deux enseignants. 
L'intérêt de la méthode réside dans le fait que les professeurs observés deviennent les observateurs et les interprètes de leur propre activité. Cette intervention permet de mieux connaitre les difficultés et les solutions que se donnent les professeurs pour surmonter ce nouvel inconnu. C'est ce que nous tentons de montrer à travers l'étude et l'analyse de l'activité des deux enseignants, aux diverses phases du dispositif d'intervention-recherche.

\section{Résultats}

À travers le tableau ci-dessous (tableau 1) nous revenons sur les autoconfrontations simples et croisées réalisées avec les deux enseignants.

Une lecture générale du tableau montre que dans la première partie, nous revenons sur les ACS des 4 séances réalisées par Thomas en évoquant les moments-clés de la mise en place du débat d'idées, son appropriation et le devenir de cet artefact dans l'activité de cet enseignant. Dans une deuxième partie, nous revenons sur les ACS avec Bernard portant toujours un même regard à l'activité enseignante à l'égard de l'artefact. Une dernière partie du tableau où chaque enseignant revient sur l'activité de son collègue. Pour une lecture plus approfondie, veuillez consulter le tableau suivant. 
Analyse ergonomique de l'activité enseignante au cours d'un cycle de football, en milie...

Tableau 1 - Présentation des autoconfrontations simples (ACS) et croisées (ACC) avec les enseignants 
Actes de la 11ème Biennale de l'ARIS : Former des citoyens physiquement éduqués. Axe 2 - Vers une éducation physique de qualité, 10.25518/sepaps20.439

\begin{tabular}{|c|c|c|c|}
\hline ACS & Enseignant & $\begin{array}{l}N^{\circ} \\
\text { Séance }\end{array}$ & Résultats selon les vignettes des autoconfrontations \\
\hline \multirow[t]{4}{*}{ ACS } & \multirow[t]{4}{*}{$\begin{array}{l}\text { Thomas } \\
\text { (Marseille) }\end{array}$} & S1 & $\begin{array}{l}\text { On constate dès le départ la motivation de Thomas à mettre en place le débat d'idée. } \\
\text { Pour lui, le débat d'idées est un cours dont il a entendu parler et que certains collègues le mettent en } \\
\text { place dans leur enseignement : « Heu... débat d'idées, c'est que c'est quelque chose que, moi (pointe le } \\
\text { doigt vers lui), j'ai déjà entendu... heu, à la faculté, avec heu... avec certains formateurs et... j'suis pas } \\
\text { sûr». } \\
\text { La mise en place des fiches (schéma du terrain) pour aider les ces élèves à s'exprimer. }\end{array}$ \\
\hline & & S2 & $\begin{array}{l}\text { Nous constatons directement que Thomas voulait recadrer dès le départ ses différentes phases, y } \\
\text { compris celle du débat d'idées. (En mettant en place une nouvelle répartition des équipes par rapport } \\
\text { au genre) }\end{array}$ \\
\hline & & S3 & $\begin{array}{l}\text { Durant la troisième séance, Thomas ne va pas installer le DI entre les deux mi-temps mais réunir tous } \\
\text { les élèves en même temps après les avoir fait jouer : " (C) : “Alors, là, vous passez directement aux mi- } \\
\text { temps des garçons." (T) : “Ouais." (C) : "Sans avoir fait..." (T) : “Débat d'idées.”» Dans ce cadre, on } \\
\text { constate que petit à petit, l'intérêt de Thomas bascule totalement vers son activité et son vrai métier de } \\
\text { professeur d'EPS. (Selon cet enseignant, être en métier, c'est lorsque les élèves sont en pratique réelle) }\end{array}$ \\
\hline & & S4 & $\begin{array}{l}\text { Thomas revient sur la mise en place du débat, mais en s'intégrant totalement dans cette phase de } \\
\text { verbalisation et en respectant les normes déjà négociées dès le début du cycle. C'est-à-dire qu'il met en } \\
\text { place le DI mais selon ses propres normes, ce qui lui garantit, à la fin, une bonne maîtrise de sa classe et } \\
\text { lui donne le sentiment de réaliser vraiment son activité et son métier d'enseignant. }\end{array}$ \\
\hline \multirow[t]{2}{*}{ ACS } & \multirow[t]{2}{*}{$\begin{array}{l}\text { Bernard } \\
\text { (Nice) }\end{array}$} & $\begin{array}{l}\text { S1 } \\
\text { S2 }\end{array}$ & $\begin{array}{l}\text { Lors de la mise en place du débat d'idées par Bernard, durant la première et la deuxième séance, nous } \\
\text { constatons que pour lui, cet artefact, qu'il prenne la forme d'une concertationZ avec implication totale } \\
\text { de l'enseignant ou non, est important du point de vue de l'activité. Mais aussi, ces moments de } \\
\text { verbalisation l'aident à développer un lien plus étroit avec ses élèves, ce qui lui garantit le bon } \\
\text { fonctionnement de son activité : « Ces moments-là heuu... de discussion... me permettent d'identifier le } \\
\text { niveau d'apprentissage de mes élèves... mais aussi, je me sens en activité et je développe un lien fort } \\
\text { avec eux. » À ce stade-là, Bernard parle de l'importance de ces moments d'échanges verbaux et de la } \\
\text { façon dont cet artefact devient un instrument sur lequel il s'appuie pour développer son activité, même } \\
\text { s'il est toujours hors des normes du DI. }\end{array}$ \\
\hline & & $\begin{array}{l}\text { S3 } \\
\text { S4 }\end{array}$ & $\begin{array}{l}\text { Revenant sur l'activité de Bernard réalisée pendant la } 3^{\mathrm{e}} \text { et la } 4^{\mathrm{e}} \text { séance, nous remarquons que durant } \\
\text { ces deux leçons d'EPS, il ne recourt pas au débat d'idées selon les normes discutées avant l'entame du } \\
\text { cycle : « (C) : “Et là, vous n'avez pas utilisé le débat d'idées ?...” (B) : “Ah ouais non [...] à part dire 'bon } \\
\text { c'est joué peut-être pas suffisamment collectif ou t'as pas levé la tête à tel moment, t’as pas servi ton } \\
\text { partenaire...””. Par la suite, Bernard développe un discours met en avant le potentiel biphasé (sens et } \\
\text { efficience) de la mise en place de l'artefact pendant les séances précédentes (séances } 1 \text { et 2). Bernard } \\
\text { dévoile encore une fois son auto-affectation en exprimant des sentiments de déception et } \\
\text { d'insatisfaction : « Là aussi, le débat d'idées, quand on en a fait un ou deux et qu'on s'est rendu compte } \\
\text { que c'est pas très productif... ça sert à rien de le faire à tout prix (grimace d'insatisfaction), s'entêter à } \\
\text { les réunir, bon (grimace).» Cette réflexion montre que cet enseignant ne trouve pas de sens à l'objet de } \\
\text { l'artefact, qui consiste à faire apprendre les élèves à travers un débat, en échangeant par exemple sur la } \\
\text { tactique, le jeu et la création de nouvelles stratégies. }\end{array}$ \\
\hline
\end{tabular}


Analyse ergonomique de l'activité enseignante au cours d'un cycle de football, en milie...

\begin{tabular}{|l|l|l|l|}
\hline ACC & Thomas & $\begin{array}{l}\text { Bernard confirme ce qu'il vient de manifester dans les ACS. Dans ce cadre, et au moment de la mise en } \\
\text { place du débat d'idées, Bernard montre qu'il est moins exigeant sur ce que produisent ses élèves } \\
\text { pendant les échanges verbaux, malgré l'importance qu'il accorde à ces moments en raison du lien qu'il } \\
\text { développe avec eux, d'un côté, mais aussi du déroulement de son activité, de l'autre. }\end{array}$ \\
Bernard & $\begin{array}{l}\text { Revenant sur le moment du débat d'idées avec Thomas, ce dernier, explique qu'il doit être présent avec } \\
\text { ses élèves pour l'animer, mais en même temps, il met l'accent sur son dilemme par rapport au DI : » } \\
\text { Largument de Thomas est que ce DI l'éloigne de ses élèves qui sont principalement en situation de jeu, } \\
\text { où il se sent plus en activité que dans l'ingénierie didactique mise en place. }\end{array}$ \\
\hline
\end{tabular}

\section{Discussion}

Tout d'abord, Thomas montre sa motivation à mettre en place l'artefact verbal. Dans un premier temps, il rencontre des difficultés diverses sur le plan didactique révélant une impuissance et une inefficacité et manifestant une activité empêchée malgré ses tentatives de départ de tout maîtriser afin de bien « faire la classe ». Ceci nous renvoie au réel de l'activité que les enseignants cherchent à atteindre sans y parvenir (Clot et al, 2000). Au fil des séances, Thomas modifie sa stratégie de mise en place du débat d'idées, dans l'objectif d'exercer son vrai métier d'enseignant d'EPS. Ensuite, il cherche à stabiliser les fonctionnalités didactiques (mésogenèse, topogenèse et chronogenèse) pour accomplir sa mission telle qu'elle lui est prescrite. Nous constatons, chez Thomas, une dynamique topogénétique qui semble plus ou moins ouverte à la participation des élèves. Malgré plusieurs tentatives pour leur déléguer ces moments, il n’hésite pas à reprendre le discours en main et à diriger le débat. Cela illustre une dynamique topogénétique avortée chez cet enseignant (Arnaud-Bestieu, 2013).

Au niveau de la dynamique mésogénétique, la recherche de Schubauer-Leoni, Leutenegger, Ligozat et Flückiger (2007) révèle que l'action du professeur dépend d'une intention conjointe qui se manifeste par une causalité d'objectifs divers pour les élèves. Si nous transposons ces réflexions au cas de Thomas, qui met en place des fiches pour mieux organiser le déroulement du DI, nous nous rendons compte que les élèves ne les utilisent pas, mais que lui s'en sert parfois. De plus, on observe que ces fiches ne sont plus employées lors des trois dernières séances. Sur ce point-là, Thomas montre, à travers les ACS, qu'il se rend compte que les élèves n'utilisent pas vraiment les fiches ; c'est pour cela qu'il ne les propose pas au cours des deux dernières séances. Dans ce cadre, il y a une tension, chez l'enseignant, entre ce qu'il croit faire avec ses fiches et leur disparition à partir de la deuxième séance et jusqu'à la dernière.

Concernant la dynamique chronogénétique, nous observons que Thomas choisit une répartition différente entre la première séance du cycle et les autres. Ce nouveau dispositif, qui différencie les filles et les garçons, engendre un potentiel plus pertinent sur le volet éducationnel, pour les élèves comme pour l'enseignant. En effet, cette nouvelle disposition des élèves rend le DI plus «économique » en termes de temps et d'apprentissage (Tiberghien, Malkoun, Buty, Souassy et Mortimer, 2007).

Avec Bernard, la mise en place du débat d'idées, son appropriation, son partage avec les élèves et son devenir sont totalement différents du cas précédent. En effet, l'enseignant, qui exerce son métier dans le milieu ordinaire à Nice, exprime dès le départ que cet artefact est un dilemme biphasé (sens et efficience). Il se limite à faire verbaliser ses élèves sans les intégrer dans le discours, afin de ne pas gêner son activité d'enseignant.

\section{Conclusion}

$\mathrm{Au}$ terme de cette recherche, il convient d'évoquer la contribution qu'elle peut apporter à la compréhension de 
Actes de la 11ème Biennale de l'ARIS : Former des citoyens physiquement éduqués. Axe 2 - Vers une éducation physique de qualité, 10.25518/sepaps20.439

l'analyse de l'activité enseignante, en mobilisant un artefact didactique (DI) cité par plusieurs chercheurs, dont nous faisons partie.

Nous pouvons dire, en guise de conclusion de cette discussion, qu'il existe une différenciation au niveau de l'instrumentalisation du débat d'idées (Rabardel, 1995) par les deux enseignants, qui est manipulé uniquement du point de vue didactique depuis son apparition il y a vingt ans. Avec le premier enseignant, le débat d'idées a retrouvé plus ou moins sa robustesse en tant qu'artefact instrumentalisé dans le développement de l'activité enseignante. $A$ contrario, ce moment discursif n'a pas résisté lors des enseignements du deuxième enseignant.

1 Dialogue $n^{\circ} 147$ - Enseignants : le travail sur le métier - janvier 2013.

$\underline{2}$ Milieu ordinaire ou difficile.

$\underline{3}$ Le débat d'idées selon les travaux de Deriaz, Poussin et Gréhaigne (1998)

4 Le football.

$\underline{5}$ Le premier enseignant appartient à un milieu d'éducation prioritaire et le deuxième à l'éducation ordinaire.

$\underline{6}$ Il s'agit d'un prénom modifié de l'enseignant.

Z Ici, l'enseignant montre qu'il préfère le terme « concertation » ou « discussion » à celui de « débat d'idées ». Même si l'appellation ne pose pas...

\section{Bibliographie}

Arnaud, P. (1989). Contribution à une histoire des disciplines d'enseignement : la mise en forme scolaire de l'éducation physique. Revue française de pédagogie, 89, 29-34.

Arnaud-Bestieu, A. (2013). Analyse des dynamiques topogénétiques en Clis: de la peur de l'échec à la dévolution avortée. La nouvelle revue de l'adaptation et de la scolarisation, (4), 241-254.

Bruner, J.S., \& Bonin, Y. (1996). L'éducation, entrée dans la culture : les problèmes de l'école à la lumière de la psychologie culturelle. Paris : Retz.

Clot, Y., Faïta, D., Fernandez, G., \& Scheller, L. (2000). Entretiens en autoconfrontation croisée : une méthode en clinique de l'activité. Perspectives interdisciplinaires sur le travail et la santé, 2(1). https://doi.org/10.4000/pistes.3833

Deriaz, D., Poussin, B., \& Gréhaigne, J.-F. (1998). Le débat d'idées. Éducation physique et Sport, 273, 80- 82.

Faïta, D., \& Vieira, M. (2003). Réflexions méthodologiques sur l'autoconfrontation croisée. DELTA : documentação de estudos em lingüística teórica e aplicada, 19(1), 123-154.

Laurent, Y., \& Saujat, F. (2014). La co-analyse de l'activité entre formateurs et formés comme révélateur de conditions à la transformation des pratiques de formation. In L. Paquay, M. Altet, M. Desjardins, J. Etienne \& P. Perrenoud (Eds.), Travail réel des enseignants et formation: quelle référence au travail des enseignants dans les objectifs, les dispositifs et les pratiques (pp. 205-218). Bruxelles : De Boeck.

Rabardel, P. (1995). Les hommes et les technologies. Approche cognitive des instruments contemporains. Paris : Armand Colin.

Roy, P., \& Tessier, D. (2006). Enseigner le football en EPS. Paris: Revue EPS. 
Analyse ergonomique de l'activité enseignante au cours d'un cycle de football, en milie...

Saujat, F. (2002). Ergonomie de l'activité enseignante et développement de l'expérience professionnelle : une approche clinique du travail de professeur (Doctoral dissertation, Aix-Marseille 1).

Saujat, F., \& Serres, G. (2015). L'activité de l'enseignant d'EPS entre préoccupations et « occupations » : un point de vue développemental. eJRIEPS. Ejournal de la recherche sur l'intervention en éducation physique et sport, 34, 4-30. https://doi.org/10.4000/ejrieps.1702.

Schubauer-Leoni, M.-L., Leutenegger, F., Ligozat, F., Flückiger, A. (2007). Un modèle de l'action conjointe professeurélèves; les phénomènes qu'il peut/doit traiter. In G. Sensevy \& A. Mercier (Eds.), Agir Ensemble. L'action didactique conjointe du professeur et des élèves dans la classe (pp. 51-91). Rennes : Presses Universitaires de Rennes.

Tiberghien, A., Malkoun, L., Buty, C., El Souassy, N., \& Mortimer, E. (2007). Analyse des savoirs en jeu en classe de physique à différentes échelles de temps. In G. Sensevy \& A. Mercier (Eds.), Agir ensemble. L'action didactique conjointe du professeur et des élèves (pp.73-98). Rennes : Presses Universitaires de Rennes.

Wisner, A. (1995). Réflexions sur l'Ergonomie. Toulouse : Octarès.

\section{Notes}

1 Dialogue $n^{\circ} 147$ - Enseignants : le travail sur le métier - janvier 2013.

$\underline{2}$ Milieu ordinaire ou difficile.

$\underline{3}$ Le débat d'idées selon les travaux de Deriaz, Poussin et Gréhaigne (1998)

$\underline{4}$ Le football.

$\underline{5}$ Le premier enseignant appartient à un milieu d'éducation prioritaire et le deuxième à l'éducation ordinaire.

6 Il s'agit d'un prénom modifié de l'enseignant.

Z Ici, l'enseignant montre qu'il préfère le terme « concertation » ou « discussion » à celui de « débat d'idées ». Même si l'appellation ne pose pas de problème, l'implication dans ces moments pour Bernard ne colle pas avec les normes établies par J-F. Gréhaigne et son équipe concernant le DI.

PDF automatiquement généré le 2023-04-26 14:28:31

Url de l'article : https://popups.uliege.be/sepaps20/index.php?id=439

Publié par ULiège Library en Open Access et distribué suivant les termes et les conditions de la licence CC-BY (https://creativecommons.org/licenses/by/4.0/deed.fr) 